\title{
DIGITAL SUPPORT SYSTEM FOR FULL-TIME AND REMOTE MASS INDIVIDUALIZED EDUCATION WITH ELEMENTS OF MACHINE LEARNING
}

\author{
Alexandr Chirtsov \\ Saint-Petersburg Electrotechnical University ETU "LETI", Russian Federation \\ Vladimir Mikushev \\ Saint-Petersburg State University, Russian Federation
}

\begin{abstract}
The requirements to the digital support system for opened individualized learning are formulated in the conditions of accelerated transition to remote forms. A version of a digital support system for development, search and provision of students with electronic educational resources in physics focused on the formation of individual trajectories of students in the educational space is considered. The possibility of several options for such a construction is allowed: 1) independent choice of the student; 2) the appointment of the trajectory by the teacher, supervisor or employer; 3) automated construction of trajectories by the system, organized on the basis of machine learning and artificial intelligence technologies.
\end{abstract}

Keywords: distance learning, educational paths, individualized learning, MOOC technologies, online education.

\section{Introduction}

The coronavirus pandemic in 2020 was a powerful accelerator of the process of computerization of education and the introduction of electronic and remote forms of subject learning (Government of Saint Petersburg, 2020). An unexpected testing ground for remote education ideas and projects in the real everyday learning process has demonstrated a number of important features of remote learning implementation in modern reality, which are necessary for further effective development of digital support tools for subject-based learning.

First of all, it turned out that the vast majority of teachers refused to use the completely acceptable in a force majeure situation and officially provided opportunity to minimize their own efforts by redirecting students to ready-made and published on-line courses. In the new conditions, the educational process in physics was mainly implemented in accordance with the established educational traditions of educational institutions, their own programs of disciplines and pedagogical ideas of teachers. At the same time, it was not the ideology and 
content of the courses that changed significantly, but their technical implementation, which required serious additional efforts from teachers due to the huge (several times) increase in labour intensity to maintain the quality of education when switching to the remote version. The difficulties encountered were mainly due to a significant reduction in the width of information communication channels between teachers and the audience of students. Interesting and important was the fact that most teachers do not use specialized electronic systems for organizing subject learning (Moodle (Büchner, 2016) or similar systems), but rather more familiar to them and students, and significantly more flexible general-purpose tools: social networks, public remote communication tools Skype (Skype, 2020), Zoom (Skytech, 2020), text, graphics, audio and video editors, etc. The practice of mass remote learning in 2020 (using the example of teaching physics courses in several Russian universities) has shown that there is a real demand from teachers not for readymade full remote courses, but for separate "point-based" electronic learning resources of various types and very diverse in topics and levels of complexity.

Objectives of the study under discussion are:

1) development of the current version of the electronic system for the accumulation, systematization and storage of electronic educational resources in order to provide teachers with convenient services for the rapid development of their own MOOC courses with partial use of information modules already available in the digital educational space;

2) testing the idea of using capabilities of digital educational tools for the automated organization of individual educational trajectories of students on the example of multilevel teaching in physics;

3) approbation of the investigated approach in a real educational process in secondary schools, specialized physics and mathematics lyceums and in technical universities.

The object of the research is methods of intensification and optimization of the remote educational process expanding under the conditions of coronavirus infection, based on the use of machine learning and artificial intelligence technologies.

To solve the set tasks, the following methodology was chosen:

1. Creation of an original electronic support system and opened individualized training and development of electronic educational resources in the form of two subsystems: client (front-end) and server (software and hardware, back-end);

2. Implementation of the electronic part of the project was carried out using the languages: PHP, MySQL, JavaScript, AJAX, HTML, CSS;

3. Carrying out test filling with existing and created using system educational resources on the example of physics; 
4. Testing the work of electronic support for individualized training in force majeure conditions of the coronavirus pandemic;

5. Planned testing of the idea of opened individualized education in educational institutions of higher and secondary education and in the self-study system.

\section{Task Assignment and Basic System Requirements}

Another serious challenge to Russian teachers from the modern education system was clearly formed in the pre-pandemic period. It consists in the presence of very contradictory requirements for higher education institutions (and, consequently, their teaching staff) through a system of indicators of their effectiveness and success. On the one hand, there is an increasing attention of the founders to the success of students in various Olympiads and competitions at both domestic and international levels. To ensure decent performance in this area, it is obviously necessary to increase the information content, volume and intensity of teaching basic subjects of the physics and mathematics cycle (at least to the level of Russian scientific and educational schools of the 80-ies of the $\mathrm{XX}$ century). The latter is quite consistent with the desire of the overwhelming number of teachers. Enhanced programs are also attractive for well-trained and motivated students. On the other hand, the existence of certain restrictions on the number of students who are expelled, and the constantly growing gap in the levels of real performance level of graduates of various secondary educational institutions against the background of the traditional tendency to reduce classroom hours allocated to exact sciences, makes setting the goal of high-quality advanced courses very problematic.

As one of the ways out of this contradictory situation, we can consider the transition to mass individualized education, the fundamental possibility of which at the present stage of development of digital and network technologies ceases to seem utopian (Chirtsov, Nikolsky \& Kurasheva, 2019). Such a transition seems feasible without significant innovations in the organization of the educational process, the natural resistance to which on the part of the structures that accompany and control this process, would most likely lead to the loss of attractiveness of the idea of improving the quality of education for those who are now able and want to really ensure its quality in the subject area. It is really feasible to preserve the existing (focused on the average student) educational process in full-time with its addition with electronic resources for those whose objective needs in the volume and level of complexity of the proposed educational material significantly differ from the average. It seems appropriate to provide students with the widest possible range of e-learning resources of 
various levels of complexity in order to be able to form a wide set of individual trajectories that best meet the needs in each specific situation.

The problems of tracing and monitoring the progress of students in the educational space in an ideal situation should be solved by them themselves under the guidance of a scientific supervisor. However, the reality is that such patronage is sometimes very formal and the trainee is de facto left without guidance. This is especially true for students whose level differs significantly from the average, both for the worse and for the better. These considerations make it reasonable to supplement the system of individualized education with a backup system for automatically tracing the trajectories of students in the educational space, the results of which should have an exclusively recommendatory status.

Naturally, a necessary component of the organization of mass individualized education is the problem of preparing high-quality electronic content, the volume of which is obviously unrealistic for almost any modern project in the field of education. The latter means that the main part of electronic educational resources can only be produced by a distributed audience of authors who create them on their own initiative, similar to how social networks function and how Wikipedia is filled in (Dounaevsky, 2013).

This development option places high demands on ensuring the maximum comfort of a digital support system for individualized education not only for consumers of educational resources, but, which is more significant at the initial stage, for their creators as well. In today's conditions, the latter means, first of all, minimal regulation of formats, levels, styles and methodological approaches to the submission of material. For its attractiveness, the system must be as simple and transparent as possible, open to the placement of resources in it by the authors themselves on any media (allowing access to it via the Internet). It is highly desirable to supplement it with easy-to-use and flexible automation tools or partial automation of the development of original content. Two such systems for simple on-line creation of interactive computer models and interactive training tests have already been developed and put into operation (Chirtsov, Nikolsky \& Mikushev, 2019; Arzamazov, Vlasov, \& Chirtsov, 2019).

The regulation on complete transparency of resource allocation by the authors seems to be very controversial and is likely to harm the trainees. However, it should be noted that the system being created and its content is itself an Internet resource and cannot automatically go beyond the restrictive limits officially imposed on any Internet resources. Creating another version of the online catalogue of educational resources is unlikely to cause additional harm to students who already have potential access to all Internet resources. Despite this, the authors of the system do not relieve themselves of the responsibility of additional control of the resources displayed on it. In cases of 
attempts to post content that has a clear anti-scientific orientation and / or violates the norms of pedagogical, academic, and universal ethics. The system administrators reserve the right to permanently deprive a number of users of the opportunity to use the system to distribute information and fakes that are harmful to qualified training. Finally, the maximum openness of the system in terms of its content allows the placement of resources containing reviews of posted content, lists of recommended and/or categorically not recommended Internet publications for students. The authors of such reviews have opportunities for fruitful work in the field of fighting for the purity of science and education, which will be very effective if they themselves and their products manage to gain popularity.

No less important is the question of the feasibility of creating another catalogue of educational Internet resources, the number of which is quite large in the Internet, for example (Unified collection of digital educational resources, 2020; A single window for accessing information resources, 2020). However, attempts to use such collections to obtain information on a subject that allows, for example, a student of a technical University to successfully answer the question "Newton's second law" on an exam in mechanics, did not lead to noticeable success in 15 minutes of searching in the listed catalogues. Apparently, the main drawback of the listed resources for a specific subject area is due to attempts to create a single search and access system suitable for the entire repository of various resources, which is not based on any author's course.

This development - a System of Mass Individualized Learning (SMIL) in its form is an electronic catalogue of educational resources on the Internet. However, in reality, it is intended as a kind of symbiosis of electronic multimedia textbooks in physics (and, in the future, in other natural science disciplines) with tools for the authors of original courses to develop, publish and attract additional resources from their colleagues, whose choice is left to the author of the course.

The functionality of the system and its capabilities significantly depend on the status provided to its users. Currently, there are four modes of operation: "Guest", "Trainee", "Teacher", and "Administrator". It seems appropriate to give a brief description of each of them.

\section{Description of the Developed System. "Guest" and "Trainee" Modes}

The user interface of the system is an electronic card ("avatar") of the resource that contains the title, list of authors, brief description of subject content, an example image of the working screen and a button, which when pressed opens a new window and runs the selected resource, a specific location of which in the Internet is irrelevant. In addition to the specified fields, which 
are completely dependent on the author's desire to fill in (or ignore), the resource avatar contains fields that display the resource's position in the search tree of the generalized course structure (course - section - topic-lecture-question), media type (text, audio lecture, video lecture, task, animation, virtual experiment, and full course, etc.), level of complexity ("Basic requirements for applicants", "Students of physics and mathematics schools and lyceums", "Physics for technical universities", "Physics for classical universities"), and others that (or a combination of them) the system searches. You can search by user-defined keywords. The ability to fill in these features is provided to the authors of the hosted resource in the "hard form" of selecting one of the positions for each of the characteristics from the existing lists related to each other. If a resource corresponds to several positions in the course navigation logic, the SMIL may contain several avatar cards that refer to the same resource, placed in the branches of search trees that correspond to the resource's characteristics. Each of the lists has a field "Outside the classifier", which contains electronic resources of authors who do not want to further clarify the position of their development in the classifier system, or if it is impossible to position the development, or because of incomplete search trees.

The system allows placement of resources both with free access and with access in agreement with the author and/or after payment (provided that the author solves all the problems of ensuring the legality of receiving income). The corresponding information is displayed in a special field located in the same block as the "Status" field, which serves to display official recommendations for using the resource in education (if any).

In addition, to the listed information about the system resources, there are a number of services that provide friendly support for the individual learning process both by the authors of the resources and by the system itself. The author's support is provided by placing links on the avatar cards that provide access to the most recommended resources of higher or lower levels of complexity, as well as to resources of the corresponding level of the card, the study of which (in the author's opinion) should precede working with the selected material. The described mechanism is most convenient when passing linear courses, in often implemented cases of students' trust in their chosen teacher and readiness to follow the path recommended by them, limited only to individual adjustment of the complexity and information saturation of the material being studied. Students who are inclined to take more active actions in tracing their trajectories in the space of educational resources can use the recommendations of their chosen authors, presented in the video provided by the card format with a list of links to similar or close resources selected by the avatar author, as well as active links located directly in the text of the annotation to the resource. 
When forming an individual educational trajectory, an alternative to the recommendations of the author of the educational resource can be automatically generated recommendations generated by the system based on the statistics of user transitions to avatars of the following educational resources collected by avatars. Marks of educational resources provided by students, their success in promoting the educational space, and the availability of links to the recommended resource from other authors.

To strengthen the system's feedback with users when generating recommendations for choosing trajectories, it is possible to use a system of built-in interactive training tests, which supports a dialogue with the test subject, during which the trainee is provided with hints along with the ability to correct or supplement the answer. Each training resource can be supplemented with an input and output test or a set of tests. The results of input testing serve as the basis for automatic generation of recommendations for correcting the learning path by adding educational material necessary for the successful development of the selected resource. In turn, input testing of resources that complement the trajectory can serve as a basis for recommendations for further adjustment of the educational trajectory. The results of the output testing serve as a basis for the avatar program's self-assessment of the associated learning resource and the formation of an in-system rating of the student. The latter is necessary for more correct accounting of the marks of resources combined by the system given by training users.

The opportunities provided by SMIL for users with the Guest and Trainee statuses are very close and are reduced to services for searching for resources and accessing them through selected avatar cards. Unlike trainees, Guests are not given the opportunity to pass tests and, as a result, influence their ratings on the rating of resources. Guest status is granted to all system users by default.

\section{System Description. "Teacher" and "Administrator" Modes}

The status of a Teacher is provided to the SMIL user at his request by the system Administrator on the basis of the electronic educational resource (or resources) submitted by the applicant. In case of violations of academic ethics or attempts to place resources in the system that harm qualified subject training, the user may be deprived of the status of Teacher.

In addition to the avatars search services available to Guests and trainees and access to their respective resources, Teachers are given the opportunity to create avatar cards for online educational resources (their own and colleagues). It is mandatory to indicate the author and author (s) of the corresponding resource in the card. Authors are also granted the right to edit and destroy their created avatar cards. If the card is destroyed, all links made to it from other 
avatars are automatically destroyed and notifications are sent to the authors of the changed avatars.

SMIL allows placing both single avatars-links to an educational resource, and building linear or branched courses and/or sets of courses in the Internet by linking avatars of transitions using the arrows "previous resource", "next resource", "harder" and "easier" and unlimited lists of recommended transitions. The arrow navigation mechanism directs authors to create chains of logically related learning resources, up to the construction of options for completed courses (or their sections) and provides students (if they trust the author) with undoubted convenience.

The most important features of the system are the ability to view the "avatar" cards themselves as equal educational resources and place HTML texts in their resource description fields with automatically added links and control buttons. The latter, obviously, in principle, allows authors to expand the functionality of the system almost indefinitely in relation to the resources they have placed. In particular, these simple features allow authors to easily build their own courses by creating avatar pages containing tables of contents and links to other developments of the author or colleagues. It is also possible to create cards with overviews, reviews, discussions, critical speeches, etc. The "classification code" field allows you to post information about the official recommendation of the resource, provided that a copy of the corresponding document signed by the author is sent to the administration. In the absence of one, the author can request a recommendation from the resource administration. The administration reserves the right to set the "not recommended" attribute in this field with an explanation of the reasons for making such a decision and, of course, preserving the author's right to delete the resource from the system.

Responsibility for the operability of links to resources placed in the system lies with the creators of the corresponding avatar cards, data about which are also registered in the cards.

The most important feature (which is purely recommendatory for the author of the resource within the framework of the development ideology) is the connection of tests that enter this resource and complete its study the decision to pass or ignore them is made by the user (trainee) and does not affect the ability to access the resource itself. In case of a failure to pass the input test, the author has the opportunity to offer the user to get acquainted with the recommended set of resources, mastering the content of which can ensure an adequate perception of the user's chosen development. Similarly, the results of the output test allow the author to provide the student with his recommendations for further completion of the course, in-depth study of the selected section, or, on the contrary, the transition of the student to a simpler level of presentation of the material. The described service provides implicit selection and promotion of the 
most successful resources in the system and is the simplest mechanism for forming individual educational trajectories.

Each avatar card of the system automatically keeps an internal record of the number of its visits, calls to the associated resource, the user's working time with the resource, the number and results of testing, the quality of the resource by users from different groups of success in passing tests, and others. These internal characteristics of resources are intended for determining their internal (available to authors) ratings in the system, which, together with the results of testing, will be used by the system to automatically generate variants of individual trajectories. Algorithms and target functions of a self-learning system for automatic tracing of educational trajectories can and should be discussed during this year, which is necessary for the initial content filling of the system.

The project administration takes over the functions of providing users with statuses in the system at their request, and if necessary, changing these statuses. Extensions of branches and sections of search trees in accordance with the availability of resources that are not included in its logic and based on the results of consultations and/or discussions with active authors are also available only to system administrators. The implementation of these features is provided by programs for editing user statuses and structures of academic disciplines.

\section{Conclusion}

Initial system filling is underway and will be completed by May 2021. There are already created original courses in classical and relativistic mechanics ( 2 and 3 level versions), classical electrodynamics (3 and 4 level versions), Optics (3 level version) and microworld physics (2 and 4 level versions). For three months of testing the system functioning, online courses have been developed, according to which training is already underway under the conditions of a massive transition to remote forms.

We would like to invite all teachers who are interested in high-quality teaching of physics, and students who want to get a competitive education in the field of exact sciences to the created resource as users of the posted information and authors-developers of their own original content. Access to the resource is open and without registration via the link http://www.physicsleti.ru/tuteline. 


\section{References}

A single window for accessing information resources. (2020). Catalog. [Electronic resource]. Retrieved from http://window.edu.ru/catalog/.

Arzamazov, N.A., Vlasov, A.P., \& Chirtsov, A.S. (2019). Development of a smart testing system to support independent work of students in self-preparation for attestations. In the collection of works of the XV International Conference, FSSO-2019, 305-307.

Büchner, A. (2016). Moodle 3 Administration. Third Edition. Packt Publishing.

Chirtsov, A.S., Nikolsky, D.Yu., \& Kurasheva, S.A. (2019). MOOK technologies as a base for solving the relevant problem of transition to mass individualized physical education: problems and their proven solutions. Computer tools in education, 1, 68-78.

Chirtsov, A.S., Nikolsky, D.Yu., \& Mikushev, V. M. (2019). JAVASCRIPT-interactive computer model generator for remote courses (MOOK) in physics. In the collection of works of the XV International Conference, FSSO-2019, 399-403.

Dounaevsky, H. (2013). Building Wiki-history: between consensus and edit warring. In Ellen Rutten, Julie Fedor \& Vera Zvereva. Routledge (Eds.), Memory, Conflict and New Media: Web Wars in Post-Socialist States.

Government of Saint Petersburg. (2020). Order of the Committee on education of 29.04.2020 No. 1033 "On the organization of activities of educational institutions in St. Petersburg". [Electronic resource] Official Internet portal of legal information. Retrieved from <http://publication.pravo.gov.ru/Document/View/7801202005070005>.

Skype (2020). The "Meet Now on Skype" function. [Electronic resource] Microsoft. Retrieved from https://www.skype.com/ru/.

Unified Collection of digital educational resources (2020). Catalog. [Electronic resource]. Retrieved from http://school-collection.edu.ru/catalog/.

Skyteach. (2020). Z00M is a platform for conducting on-line classes. [Electronic resource]. Retrieved from https://skyteach.ru/2019/01/14/zoom-platforma-dlya-provedeniyaonlajn-zanyatij/. 